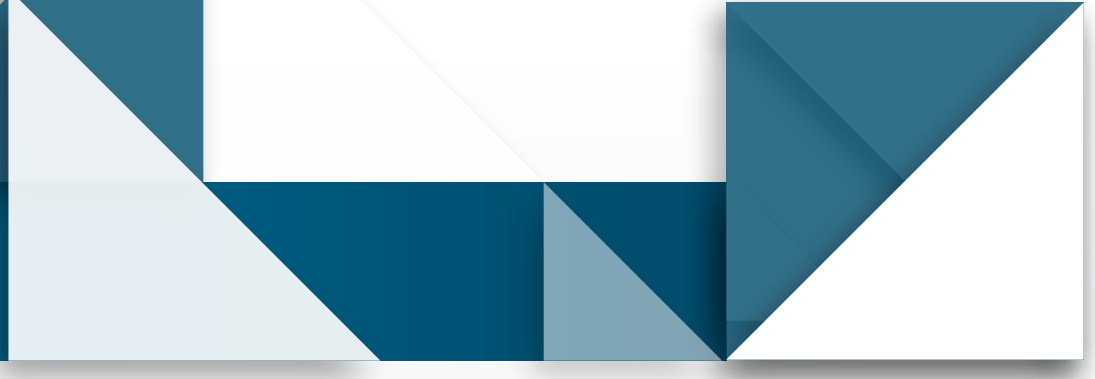

\title{
Conocimiento didáctico del 1 contenido en la enseñanza del campo eléctrico
}

- Pedagogical Content Knowledge on Electric Field Teaching

- Conhecimento Didático de Conteúdo no Ensino de Campo Elétrico

\section{Jaime Duván Reyes Roncancio* Carmen Alicia Martínez ${ }^{* *}$}

\section{Resumen}

Este artículo presenta resultados de investigación correspondientes a la tesis doctoral en educación en ciencias titulada "Conocimiento Didáctico del Contenido (CDC) en profesores de física en formación inicial: el caso de la enseñanza del campo eléctrico". El enfoque metodológico cualitativo utilizado fundamentó la aplicación de una encuesta y una entrevista semiestructurada, así como el análisis de contenido del plan de aula de un profesor de física en el prácticum. La triangulación de la información obtenida a partir de estos instrumentos permitió constituir el caso de la enseñanza del campo eléctrico desde la caracterización del CDC del profesor de física, proceso en el cual se utiliza como referencia una matriz estilo hipótesis de progresión y el uso de metáforas.

\footnotetext{
Abstract

This paper presents the results obtained from a doctoral thesis on Science Education entitled "Pedagogical Content Knowledge (PCK) in pre-service physics teachers: "the teaching electric field case" The qualitative methodological approach used allowed the application of a survey, a semi-structured interview and a content analysis of a lesson plan, all from a pre-service physics teacher during his practicum. Triangulation of information obtained from these instruments helped us characterize the PCK of teaching electric field, a process in which a progression hypothesis and the metaphors were used as reference.
}

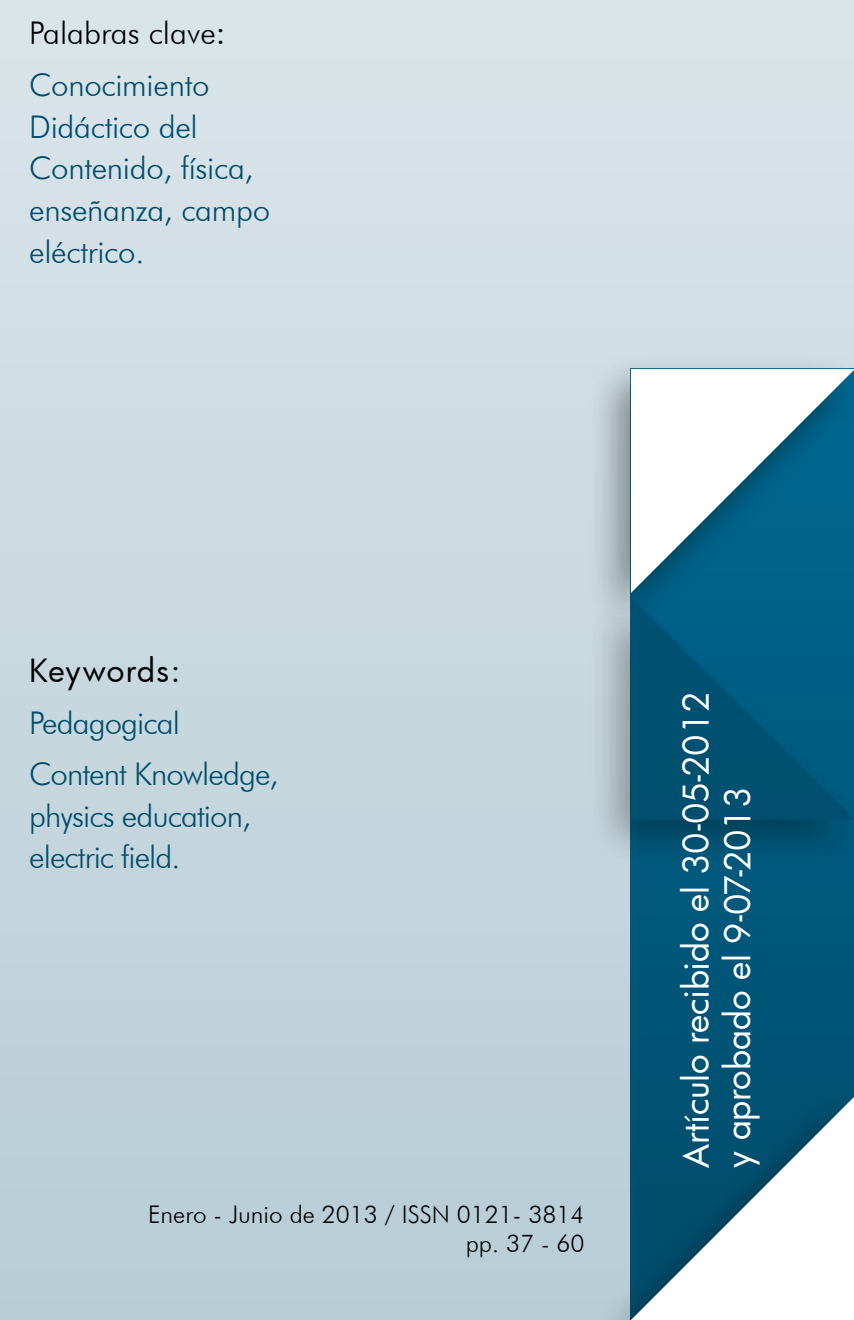

Doctorado interinstitucional en Educación Universidad Distrital Francisco José de Caldas: jdreyesr@udistrital.edu.co

** Doctorado interinstitucional en Educación Universidad Distrital Francisco José de Caldas: camartinezr@udistrital.edu.co

Didáctico del

Contenido, física

enseñanza, campo

eléctrico.

Keywords:

Pedagogical

Content Knowledge,

physics education,

electric field. 


\section{Resumo}

Este artigo apresenta resultados de investigação que correspondem à tese de doutorado em Educação em Ciências intitulada "ConocimientoDidácticodelContenido (CDC) em profesores de física em formación inicial: el caso de laenseñanzadel campo eléctrico". O enfoque metodológico qualitativo utilizado fundamentou a aplicação de um questionário e uma entrevista semi-estruturada, bem como a análise de conteúdo do plano de aula de um professor de física em exercício. A triangulação da informação obtida por meio desses instrumentos permitiu construir o caso do ensino de Campo Elétrico

Palavras-chave: Conhecimento Didática de Conteúdo, Física, Ensino, Campo Elétrico. a partir da caracterização do CDC do professor de Física, processo no qual se utiliza como referência uma matriz do estilo hipótese de progressão e o uso de metáforas. 


\section{Introducción}

El Conocimiento Didáctico del Contenido (CDC) como constructo académico (Shulman, 1986; Grossman, 1989 y Abell, 2008) se constituye en alternativa investigativa que asume el trabajo del profesor desde una perspectiva profesional, pero que llama la atención sobre los procesos de transformación de la materia en conocimiento de la materia para la enseñanza. Para el caso de este documento, los desarrollos específicos del CDC en profesores de física se han venido abordando de manera incipiente, y por demás aislada. Frente a esto, la tesis doctoral asume un papel protagónico al investigar el CDC a partir de cuatro de sus componentes: los contenidos, las ideas de los estudiantes, las actividades y la evolución. Este proceso atiende la pregunta fundamental de investigación: ¿̇cómo caracterizar el CDC de un profesor de física en formación inicial en la enseñanza del campo eléctrico en el bachillerato? La investigación se constituyó metodológicamente desde los principios de la investigación cualitativa de orden interpretativo, enfoque desde el cual se configuraron tanto los tres instrumentos utilizados como la perspectiva de análisis de contenido utilizada (Bardin, 1986)

\section{Marco teórico}

Los estudios iniciales sobre conocimiento del profesor (Grossman, Wilson y Shulman, 1989) cuestionaron el conjunto de investigaciones de proceso-producto que les precedían, pues estas proponían indagar el conocimiento de los profesores en relación exclusiva con el rendimiento de los alumnos. En este sentido, Shulman (1986) comienza a posicionar una propuesta alternativa en el marco de lo que denomina como el "paradigma perdido" en la investigación proponiendo:
Dentro de la categoría conocimiento

didáctico del contenido incluyo los temas más comúnmente enseñados en una determinada asignatura, las formas más útiles para representar las ideas, las analogías, ilustraciones, ejemplos, explicaciones y demostraciones más poderosas, en una palabra, las formas de representar y formular el contenido para hacerlo comprensible a otros (Shulman, 1986, p. 9).

Si bien las investigaciones sobre CDC reconocen esta definición de Shulman en su sentido histórico y propositivo, también han venido aportando resultados tanto en su caracterización (Van Driel, 1999 y Abell, 2008) como en su composición (ver tabla 1).Un aspecto fundamental del constructo CDC se encuentra en su ubicación dentro del contexto del conocimiento del profesor desde una perspectiva profesional (Marcelo, 1992; Bolívar, 1993; Benejam, 1993; Garritz, Trinidad y Velasco, 2004; Valbuena, 2007; y Reyes, 2010).

Con todo, los investigadores en general han posicionado la discusión sobre el CDC no solo en el marco de la práctica profesional de docentes en ejercicio, sino que también muestran una preocupación por los procesos de formación del profesor, especialmente al cuestionar las tendencias que validan una formación profesional que hace poco énfasis en conseguir que los profesores en formación piensen sobre la materia que han de enseñar en términos de sus contenidos didácticos (Gudmundsdottir y Shulman, 1990, p.p.33). Esto, por un lado, permite discutir la posibilidad de caracterizar el CDC de los futuros profesores desde una perspectiva donde las didácticas específicas (Bolívar, 1993) adquieren un significado importante especialmente en relación con su necesaria diferenciación con el conocimiento pedagógico general. 


\section{Componentes del Conocimiento Didáctico de Contenido (CDC)}

En este marco general, se contextualiza el problema de la investigación de la tesis doctoral entorno a la caracterización del CDC de profesores de física en formación inicial para el caso de la enseñanza del campo eléctrico en el bachillerato. Este trabajo de caracterización se hace en concordancia con lo que evidencia la investigación en esta línea, por lo menos en lo que respecta a la enseñanza de las ciencias y los estudios de profesores en preservicio y en servicio. En este sentido, el CDC como constructo (Abell, 2008, Carlsen, 1999 y Grossman, 1990) es emergente en la enseñanza de algunas disciplinas; en particular para el caso de la enseñanza de la física y la formación de profesores, los desarrollos alcanzados (Reyes, 2010) evidencian la necesidad de continuar con estudios puntuales sobre el CDC de profesores de física en formación, principiantes y experimentados.

Tabla 1.Componentes del CDC como constructo emergente.

\begin{tabular}{|c|c|c|c|c|c|c|c|c|c|c|}
\hline & 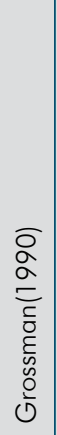 & 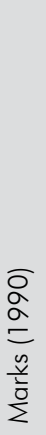 & $\begin{array}{l}\bar{a} \\
\alpha \\
\alpha \\
\bar{c} \\
\bar{d} \\
\frac{0}{2} \\
\bar{\vdots} \\
\dot{U}\end{array}$ & 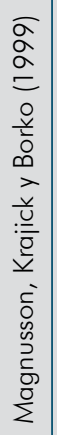 & 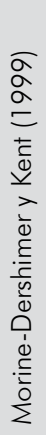 & 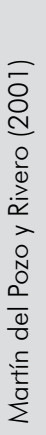 & 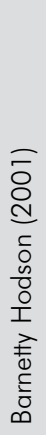 & 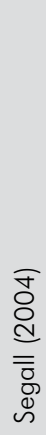 & 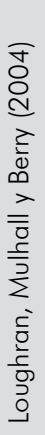 & 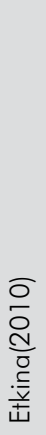 \\
\hline \multicolumn{11}{|l|}{ Currículo } \\
\hline \multicolumn{11}{|c|}{ Contenidos } \\
\hline \multicolumn{11}{|c|}{ Ideas de los estudiantes } \\
\hline \multicolumn{11}{|c|}{ Aprendizaje de los estudiantes } \\
\hline \multicolumn{11}{|c|}{ Estrategias de enseñanza } \\
\hline \multicolumn{11}{|c|}{ Evaluación } \\
\hline \multicolumn{11}{|c|}{ Propósitos de la enseñanza } \\
\hline \multicolumn{11}{|c|}{ Organización y secuenciación de lecciones } \\
\hline Contexto & & & & & & & & & & \\
\hline
\end{tabular}

Sin embargo, tal como se ilustra en la tabla 1, no hay una postura unificada en la comunidad investigativa sobre los componentes del CDC, lo que ha permitido en últimas, una flexibilidad relativa a la hora de investigar, así como de considerar el CDC en la formación de profesores. Para el caso que nos ocupa en este documento, se recoge la perspectiva general planteada por Etkina (2010), en razón a su propuesta de incluir el CDC en su programa de formación de profesores de física con los siguientes componentes: a) orientaciones hacia la enseñanza, b) conocimiento sobe el currículo en física, c) conocimiento de las ideas de los 
estudiantes, d) conocimiento de estrategias efectivas de enseñanza y e) conocimiento sobre métodos de evaluación (Etkina, 2010, p.0201 10-3). Estosretoman la propuesta de Magnusson, Krajick y Borko (1999), pero, dado el objetivo de la tesis, y en especial por su especificidad en la caracterización de la enseñanza del concepto campo eléctrico por parte de los profesores en formación, los dos primeros componentes del CDC en Etkina (2010) se pueden agrupar en uno solo, que tendrá la denominación de contenidos para la enseñanza, quedando así los siguientes cuatro componentes de referencia: a) contenidos para la enseñanza, b) actividades, c) ideas de los estudiantes $y, d$ ) evaluación.

La selección de estos cuatro componentes obedece entonces al potencial descriptivo y propositivo que tienen respecto a la caracterización del CDC para el caso concreto de estudio, su relación directa con otros componentes propuestos en esta línea de investigación (tabla 1) y la coherencia con las propuestas de formación de profesores de física que incluyen explícitamente al PCK en sus contenidos (Etkina, 2010). En adición, la perspectiva de investigación de estos componentes tiene principalmente un enfoque integrador, ya que, si bien se reconoce la necesidad de caracterizar cada uno de estos a profundidad en el caso de la enseñanza del campo eléctrico, también se asume que cada uno de estos componentes en forma aislada no es el CDC (figura 1). En lo que sigue, se presentan los fundamentos teóricos de cada uno de estos componentes, respecto a los cuales se desarrolló el proceso metodológico.

Figura 1. Componentes del CDC en el marco del conocimiento profesional del profesor.

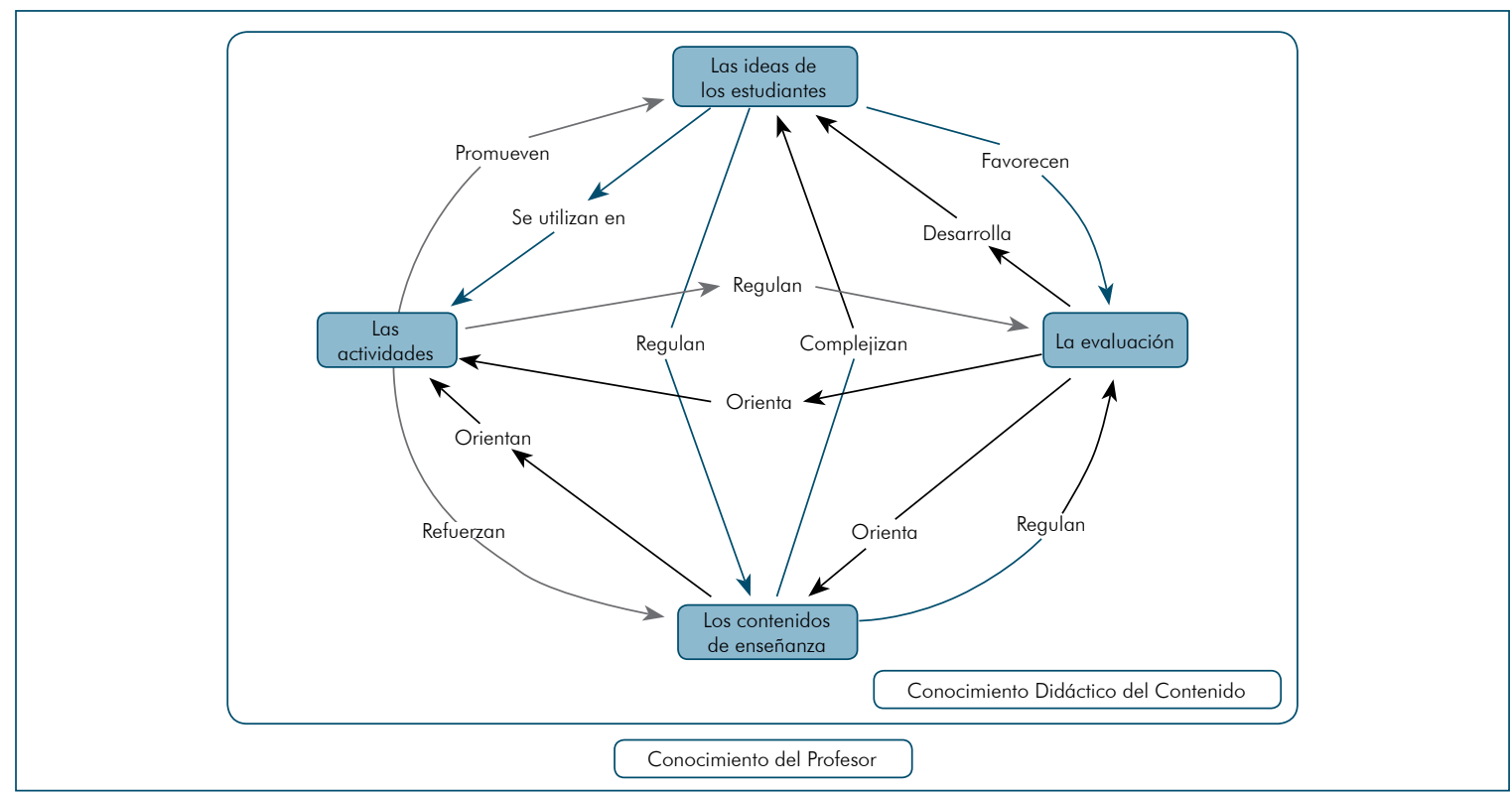

Los contenidos para la enseñanza del campo eléctrico

Este componente principalmente se refiere a los contenidos conceptuales (campo eléctri$\mathrm{co})$, los contenidos procedimentales y los contenidos actitudinales que el profesor planea y desarrolla en clase al enseñar el concepto de campo eléctrico. Al respecto, una forma muy clásica en la que se muestra el concepto de campo eléctrico en relación con otros contenidos (tanto en las clases tradicionales como en los libros de texto generalmente) no discute la naturaleza epistemológica del concepto 
ni se asoma a la necesidad de diferenciación con el concepto de fuerza, por ejemplo, cuando se encuentran definiciones del campo asociadas a la ecuación $(E=F / q)$ e inmediatamente se procede a resolver ejercicios de aplicación. Esta visión está muy ligada al peso que le da el profesor a la perspectiva newtoniana. Martín y Solbes (2001) encuentran que los profesores confunden la fuerza F con el vector intensidad de campo $\mathrm{E}$ cuando son inquiridos por dibujar este último vector en puntos donde no hay cargas eléctricas. Esta situación puede influir la selección de contenidos en el sentido de validar implícitamente la forma en que es organizada la aproximación al concepto de campo partiendo desde la idea de carga y luego de fuerza eléctrica (ley de Coulomb), cuando se insiste en este tipo de organizaciones y jerarquizaciones implícitamente se enfatiza en el tratamiento de los conceptos desde una perspectiva newtoniana, es decir, donde las interacciones eléctricas se entienden a distancia y en simultaneidad.

En general, los libros de texto (Villegas y Ramírez, 1998; Zitzewitz, Neff, Davids, 1995; Hewitt, 1999; y Wilson y Buffa, 2003) secuencian los temas como en la figura 2, y los profesores que asumen su planeación y el desarrollo de las clases en este sentido también involucran el concepto de diferencia de potencial como una aplicación del concepto de campo eléctrico, sin mediación de una reflexión sobre el concepto de energía potencial, o de una aproximación que lo diferencie de la visión newtoniana de la caída libre -donde las partículas tienen energía potencial. Esta visión tiene como base una comprensión del campo eléctrico como instrumento para analizar la interacción eléctrica, pero desde una perspectiva en la que no se diferencia entre fuerza y campo (Viennot y Rainson, 1992, y Furió y Guisasola, 1998a y 1998b) y más bien se centra en revisar si los estudiantes resuelven bien ecuaciones, o ejercicios tipo lápiz y papel donde su uso se hace necesario.

Figura 2. Secuencia de contenidos conceptuales donde seasume implícitamente la necesidad de la idea de carga y de interacciones a distancia. Acumulativa y lineal.

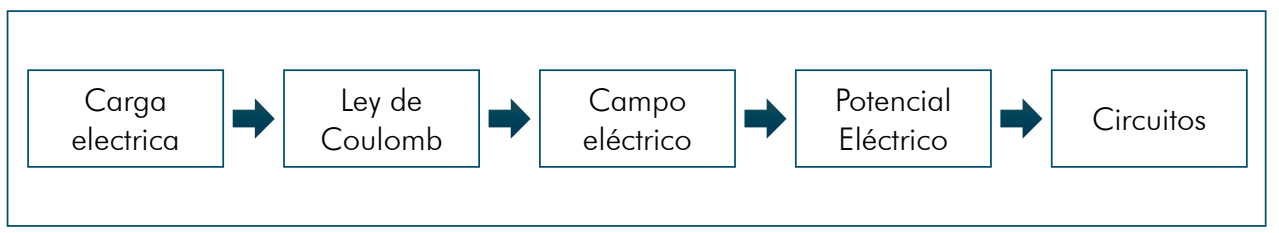

Con todo, este enfoque tampoco discute los tratamientos usuales de organización y jerarquización de los contenidos, ni considera de manera importante los conceptos de los estudiantes para abordar el tratamiento de los temas. Esto es lo que a juicio de Poon (1986) resulta cuando no se discute el significado físico del concepto y como consecuencia:"el lenguaje de campo aparenta ser algo redundante, y ofrece meramente una alternativa para expresar la ley de gravitación universal de Newton o la ley de Coulomb" (Poon, 1986, p.307). Aquí, el concepto decampo eléctrico no es organizador, sino, más bien, un elemento dentro de una máquina que organiza los contenidos en forma lineal para la 
enseñanza. Es decir, no hay valoraciones de la organización y secuenciación de los contenidos de los libros o de los planes de estudio hechos por otros.

Con esto se hace imperativo resaltar que enla base de la visión acumulativa lineal (ver figura 3) estaría la no diferenciación epistemológica entre acción a distancia y campo y, por tanto, de una falta de criterio sobre la pertinencia de ver los temas unos como prerrequisitos de otros sin mediar reflexión epistemológica. Diríamos que, en este caso, el profesor de física no se asume como sujeto-epistémico-profesor (Perafán, 2004), sino más bien como sujeto-relator-profesor. En este sentido, la exposición lineal de los contenidos se hace sin considerar los "saltos cualitativos ni los problemas que dieron lugar a nuevos conceptos de mayor poder explicativo" (Furió y Guisasola, 1997, p.264).

Figura 3. El Concepto de Campo en una Visión acumulativa lineal de organización del contenido.

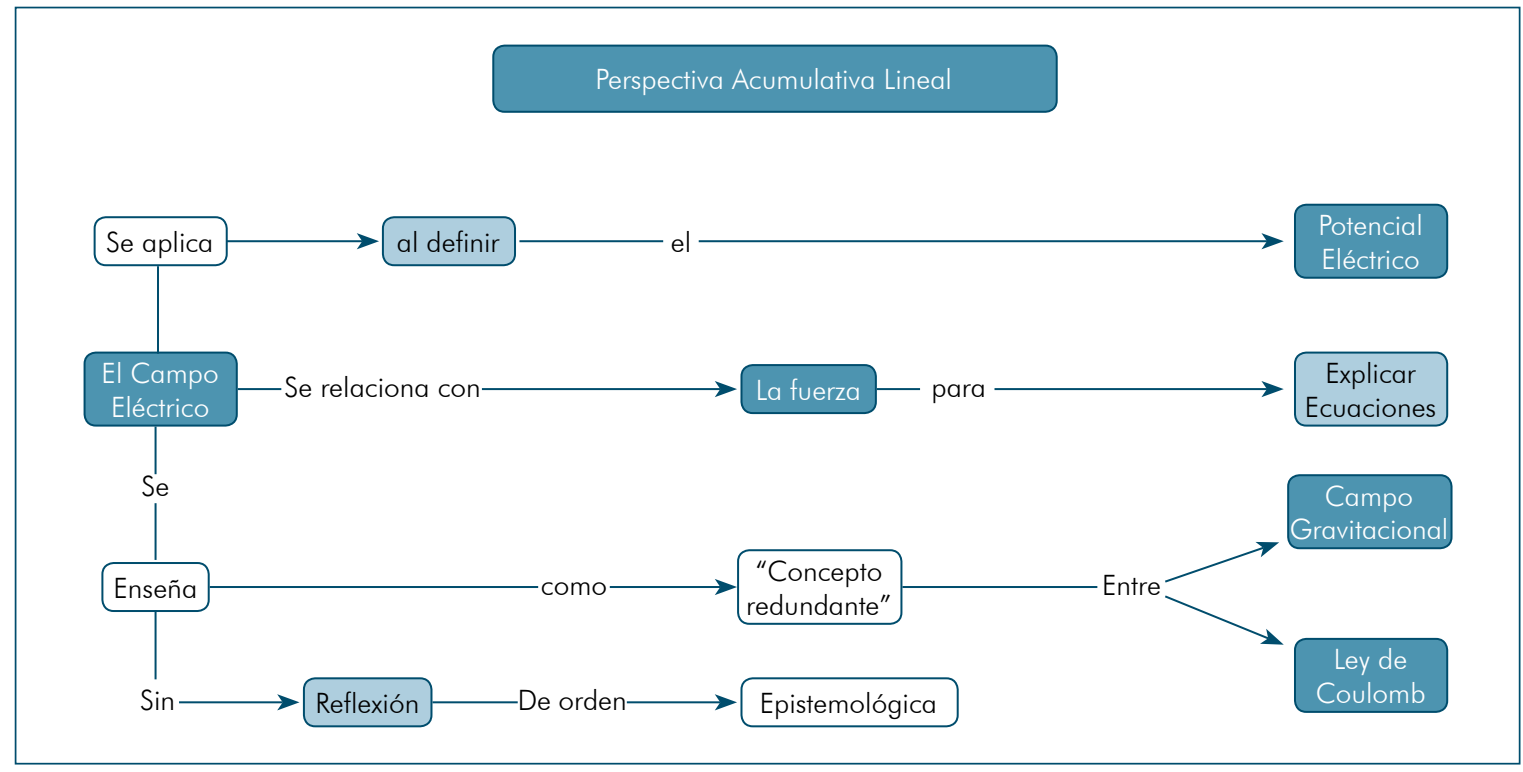

Este tipo de organizaciones pueden también entenderse como de un nivel de referencia implícito, en el que se asumen los contenidos tal como se entiende el modelo teórico de la electricidad en la actualidad, donde la idea de carga y de fuerza eléctrica parecieran cantidades físicas con significados que no necesitan ser revisados, sino transmitidos, y, por tanto, entregados en el más purista de los sentidos. El campo eléctrico, entonces, termina siendo introducido en forma "a problemática" y, por tanto, "arbitraria" (Furió y Guisasola, 1997,p.264).

Por otro lado, si se considera que la ontología del campo fundamenta su existencia, es decir, que se le da alguna realidad al campo, entonces este se constituye en referencia para la selección y jerarquización de contenidos, pues, al igual que la masa, la luz y otras realidades físicas, los contenidos asociados al campo eléctrico deberán estar en relación con la realidad que se quiere construir. En efecto, si el campo eléctrico es: "una entidad tan real como las fuentes que lo crean, realidad que resulta más evidente cuando las fuentes dependen del tiempo, ya que, entonces, el campo transporta energía y momentos, lineal y angular, como las partículas materiales" (Pomer, 1994, citado en Martín y Solbes, 2001, p. 394). 
Entonces, la planeación y secuenciación de los contenidos deberían corresponderse (por lo menos en un nivel deseable y en coherencia con esta postura) con un proceso constructivo de la realidad del campo, el cual puede tomar elementos de la historia de la física o los relacionados con psicogénesis de los conceptos científicos, especialmente relacionados con: a) la diferenciación entre fuerza y campo, b) la organización de contenidos que asocien realidad al campo en términos de acciones locales y no a distancia, c) la representación del campo a partir de situaciones experimentales y d) la discusión sobre las formas usuales de organización de los contenidos conceptuales asociados al campo eléctrico que se presentan en la enseñanza tradicional o en los libros de texto.

\section{Actividades}

El conocimiento sobre el desarrollo de las tareas y actividades de enseñanza en las que el profesor involucra a los estudiantes. Es decir, este componente se refiere a las acciones concretas que el profesor desarrolla en la clase, como ejercicios de lápiz y papel, explicaciones, representaciones, discusiones, presentaciones, exposiciones, laboratorios, talleres, socializaciones, entre otras. Törnkvist, Peterson y Tranströmer (1993) señalan que, si bien no es algo nuevo detectar dificultades de comprensión sobre los vectores como entidades matemáticas, así como las subsecuentes representaciones de diferentes conceptos físicos, una alternativa para la enseñanza de campo eléctrico podría considerar el trazado de flechas de diferente tamaño, así como la necesidad de acudir a representaciones integrales de las cantidades físicas asociadas, llamando la atención sobre la importancia de la "secuencia jerárquica de los conceptos (carga geometría-campo línea-fuerza vector-(vector velocidad)-trayectoria)"(p. 338), dado que, a su juicio, este aspecto "no se ha entendido completamente" (p. 338). Este proceso se constituye relevante en el marco del CDC del profesor de física por cuanto las actividades de representación, propias de su labor, o las asignadas o construidas por sus estudiantes pueden enriquecerse. En este punto, Arons (1990) sugiere considerar la bondad de representarlas fechas con colores diferentes en relación con las cantidades físicas.

Martín y Solbes (2001), en su propuesta alternativa para la enseñanza del campo, se basan en un enfoque constructivista y recogen tareas y actividades asociadas a la "formulación y resolución de problemas abiertos, emisión y contrastación de hipótesis" (p. 396); en concreto, distinguen productos de la investigación didáctica en relación con la "resolución de problemas, los trabajos prácticos, la evaluación de conocimientos, las interacciones ciencia-técnica-sociedad" (p. 396). Estos autores destacan una revisión bibliográfica de las estrategias que la literatura investigativa muestra en consonancia con las problemáticas de los estudiantes en la comprensión del concepto de campo, y ponen de manifiesto la necesidad de emprender tareas o actividades de enseñanza enfocadas a considerar las visiones epistemológicas subyacentes, en particular las de corte mecanicista que tienen un peso bastante grande en las explicaciones (no reflexivas) 
de la interacción eléctrica. Sugieren también que los programas de formación contribuyen con este enfoque $y$, por tanto, las actividades y tareas alternativas deben considerar esta realidad para implementar nuevas estrategias de formación.

De otra parte, un análisis del contenido de algunos libros de texto en física para la media vocacional, en lo que respecta a la electricidad, y más específicamente al campo eléctrico, evidencia una tendencia mayoritaria a sugerir la realización de ejercicios de lápiz y papel como actividad principal. Estos ejercicios se platean para entrenar en el análisis de aplicaciones de la ecuación de intensidad de campo eléctrico $(E=F / q)$ y la ley de Coulomb, principalmente, y luego para revisar la ecuación del potencial eléctrico.

Tabla 2. Tipos de actividades que algunos libros de texto de física incluyen en el tratamiento del campo eléctrico. En gris la presencia de la actividad en cada libro

\begin{tabular}{|c|c|c|c|c|c|c|c|}
\hline & 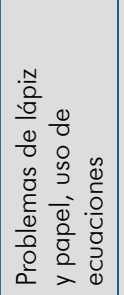 & 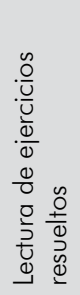 & 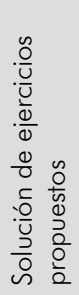 & 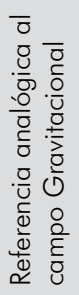 & 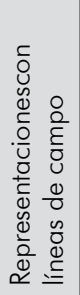 & 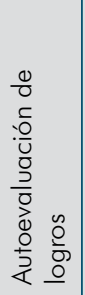 & 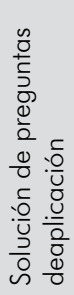 \\
\hline \multicolumn{8}{|c|}{ Zalamea, Rodríguez y Paris (2001) } \\
\hline \multicolumn{8}{|l|}{ Morales e Infante (2005) } \\
\hline \multicolumn{8}{|c|}{ Zitzewitz, Neff y Davids (1995) } \\
\hline \multicolumn{8}{|l|}{ Hewitt (1999) } \\
\hline Villegas y Ramírez (1998) & & & & & & & \\
\hline
\end{tabular}

En estos textos, generalmente, se asocia el concepto de campo eléctrico con la representación de las líneas de campo, sin mediar procesos de indagación o situaciones experimentales que inviten a elaborar argumentaciones o representaciones de este tipo. Lo que hace la mayoría de estos textos es mostrarlas líneas como representaciones ya elaboradas del campo y el estudiante tiene que aprenderse las distribuciones de las líneas, si es para placas paralelas o puntuales, según los códigos asociados a los signos de la carga. Son pues actividades cerradas en las que los estudiantes deben asumir al campo eléctrico como las líneas de fuerza, o que invitan a considerarlo desde representaciones bidimensionales tan solo a lo largo de las líneas de fuerza que se dibujan. La idea de actividad cerrada también tiene que ver con situaciones asociadas a la solución de ejercicios de lápiz y papel con única respuesta y único procedimiento. Aspecto que se puede revisar también en el tratamiento que algunos libros de texto hacen a los ejercicios de ejemplo, así como de las actividades experimentales.

\section{Ideas de los estudiantes}

Se refiere al conocimiento del profesor sobre las ideas previas o alternativas de sus estudiantes acerca del campo eléctrico y al conocimiento sobre sus dificultades en la comprensión de este. En especial, este componente se hace importante en la medida que revela la perspectiva pedagógica de la enseñanza que se asume, ya que desde donde se consideren las 
ideas de los estudiantes, así como la manera en que se conciben las actividades de exploración de estas y aquellas relacionadas con sus referencias posteriores en el desarrollo de las clases, se puede construir una caracterización del CDC del profesor en formación inicial. Etkina (2010) concibe este componente como "conocimiento de las ideas curriculares previas de los estudiantes cuando ellos están construyendo un nuevo concepto. Conocimiento de las dificultades que los estudiantes puedan tener interpretando el lenguaje de la física, dado que es diferente al lenguaje cotidiano" (p. 020110-3). Para el caso particular de la enseñanza del campo eléctrico, un aspecto relevante sobre el desarrollo de las ideas de los estudiantes consiste en las diferenciaciones que se pueden establecer entre los conceptos de fuerza, campo y energía. Al respecto, Martín y Solbes (2001) advierten como:

Durante el proceso de enseñanza se olvida, frecuentemente, que las interacciones entre partículas pueden describirse de diversas formas (fuerzas, campos, energías, etc.), y podemos desorientar a los alumnos si no se clarifican suficientemente los conceptos, estableciendo sus relaciones, sus diferencias y ámbitos de aplicación (p. 393).

Pero esto implica considerar que la abstracción inherente al concepto de campo no siempre adquiere connotaciones familiares con la cotidianidad de los estudiantes, aun cuando esta situación no es ajena para conceptos de otras áreas de la física, lo cual se constituye precisamente en un conocimiento que no necesariamente deviene tan solo de la teoría de campos, sino que también involucraría un conocimiento de teorías pedagógicas que acompañan la inclusión o detección de las ideas de los estudiantes. La posible diferenciación de estos conocimientos está asociada a los procesos de reflexión que hacen los profesores sobre sus prácticas de clase, la toma de decisiones en esta y la investigación que de esto de cuenta. Törnkvist et al. (1993) advierten sobre la importancia de considerar las ideas difusas que pueden tener los estudiantes sobre la fuerza y el campo de fuerza en relación con la manera como se dirige la enseñanza de las representaciones gráficas con conceptos asociados. Como este estudio se realiza en la universidad, se constituye en referente en cuanto se aproxima a los tipos de conocimiento de contenido que pueden influenciar la mirada de las ideas de los estudiantes en los practicantes de licenciatura. Es decir, si se considera que las ideas sobre campo eléctrico de los pupilos pueden ser estudiadas, o tenidas en cuenta en el proceso, uno de los criterios de referencia puede ser el asociado a las propias confusiones que el practicante haya tenido en su formación.

Ahora bien, las ideas de los estudiantes también pueden ser incluidas en referencia a sus concepciones sobre la fuerza y la acción a distancia o el papel del medio en la interacción. Al respecto, Martín y Solbes (2001) han identificado que "El alumno no llega a conocer las diferencias que sobre la interacción entre partículas introduce la teoría de campos mediante acciones contiguas frente a la interpretación newtoniana a través de acciones a distancia" (p. 395). Situación 
que merece la atención por cuanto posiciona en forma concreta cómo atender este tipo de dificultades desde la perspectiva del CDC. En este sentido, Bar, Zinn y Rubin (1997) encontraron en un estudio sobre ideas de los niños acerca de la acción a distancia que: cerca del $50 \%$ de los participantes sugirieron que la fuerza eléctrica necesita aire como medio conductor, lo cual, a juicio de estos investigadores, manifiesta una necesidad general de los niños por establecer una conexión entre los cuerpos. Así, las ideas de los estudiantes pueden explorarse desde perspectivas asociadas a la gravedad, la fuerza, la electricidad, la atracción, la repulsión, la conexión entre cuerpos, la necesidad de un medio. De esta manera, la idea de gravedad en los niños se asocia con características como: la presión del aire, la conducción del aire y el incremento con la altura respecto al piso (p. 1 139). En el caso de las dos primeras, los niños afirman que la gravedad no existe en el espacio o cerca de la Luna y, para el caso tercero, afirman que la gravedad se detiene cuando el aire se detiene. Respecto a la idea de fuerza, estos autores también plantean que los niños le dan un carácter protagónico al contacto entre cuerpos, de manera que se asume la acción a distancia en relación necesaria con un medio conductor o algún otro mecanismo (p. 1139).

Nótese que, en este caso, la idea de fuerza se puede asumir desde una perspectiva algo sustancialista en el sentido de la necesidad del contacto, pero esto no necesariamente surge en los estudiantes de colegio, pues aquí parece haber más elementos que van enriqueciendo las ideas de los estudiantes. Por ello, Furió y Guisasola (1998b) encontraron que los estudiantes del bachillerato, e incluso de universidad, persisten en sus explicaciones newtonianas en situaciones directamente asociadas con el campo eléctrico, es decir, los estudiantes interpretan desde la acción a distancia y no desde el papel del medio. Esto, a juicio de los investigadores, se debe principalmente a que los estudiantes no establecen una clara diferencia entre dos conceptos: a) intensidad del campo eléctrico y b) fuerza eléctrica. En cuanto a la idea de la necesidad de un medio, Furió y Guisasola (1998a y 1988b) identifican también dificultades conceptuales en los alumnos a la hora de distinguir la manera de entenderla interacción eléctrica, ya que los estudiantes (incluso los universitarios familiarizados con el electromagnetismo) continúan interpretándola desde la perspectiva colombiana. Raduta (2005) considera que esta situación se encuentra asociada a dificultades de orden epistemológico centradas en formas de razonamiento que contemplan sentido común y simple casualidad, situación que podría tener sus orígenes en las formas tradicionales como se han dictado las clases de electricidad, pues su alto sentido acumulativo de contenidos no permite análisis cualitativos profundos de estos.

Tabla 3. Los aportes de la investigación sobre ideas de los estudiantes y la enseñanza del campo eléctrico permiten distinguir algunas dificultades en su comprensión.

Ahora bien, se sabe que Viennot y Rainson (1992) han señalado la importancia de las ideas de los estudiantes sobre el campo en relación con las categorías: a) existencia de campo eléctrico solamente si hay movilidad de carga y b) sin corriente no hay campo. En estos casos, subyace una noción causal de la existencia del campo, pues, si no se notan los efectos, se asume que este no existe, c) la causa del campo está en la fórmula y tiene origen solamente en los conductores. Una síntesis de las problemáticas detectadas sobre las formas de comprensión del campo eléctrico se muestra en la tabla 3 . Ideas de los estudiantes sobre campo eléctrico 


\section{Dificultades}

"El alumno no llega a conocer las diferencias que sobre la interacción entre partículas introduce la teoría de campos mediante acciones contiguas frente a la interpretación newtoniana a través de acciones a distancia" (p. 395).

\begin{tabular}{ll}
\hline $\begin{array}{l}\text { Törnkvist, Peterson y } \\
\text { Tranströmer (1993) }\end{array}$ & Idea difusa de fuerza o de campo. \\
\hline Bar, Zinn y Rubin (1997) & La idea de interacción. \\
\hline Furió y Guisasola (1998a) & Persistencia en visión newtoniana. \\
\hline Raduta (2005) & $\begin{array}{l}\text { El papel del sentido común y la causalidad simple. } \\
\text { Visión estática del campo. }\end{array}$ \\
\hline Bagno (1986) & $\begin{array}{l}\text { Los textos imponen visiones inmutables del campo que refuerzan } \\
\text { perspectivas intuitivas. }\end{array}$ \\
\hline $\begin{array}{l}\text { Viennot y Rainson (1992) } \\
\text { y Viennot (2002) }\end{array}$ & $\begin{array}{l}\text { Enfoque cuasiestático del campo. } \\
\text { Nociones causales lineales de la existencia del campo. }\end{array}$ \\
\hline
\end{tabular}

\section{Evaluación}

El conocimiento sobre los procesos, formas y estrategias de evaluación, principalmente indagados aquí desde dos perspectivas generales: la evaluación sumativa y la evaluación formativa. Se refiere al conocimiento sobre la evaluación que subyace y se explicita en las propuestas de enseñanza y en su desarrollo. Tanto los criterios como las herramientas revelan características del CDC en el sentido de los procesos de verificación de aprendizaje que en el contexto de la enseñanza de las ciencias atiende cualquier profesor. Etkina (2010) propone que este es un conocimiento sobre los métodos de evaluación, en particular de la comprensión conceptual de los estudiantes, la resolución de problemas y en general las capacidades científicas, es también el conocimiento sobre cómo ayudar a los estudiantes a autoevaluar su trabajo y hacerlos partícipes de una reflexión significativa.

Así, la evaluación se puede entender de diversas formas, ya sea como un proceso continuo en el que el profesor desarrolla la interacción con los estudiantes y establece juicios cambiantes sobre sus aprendizajes, o como un proceso terminal en el que los juicios, ya cualitativos o cuantitativos, se generan al final del desarrollo temático y experimental (si es el caso). Geli (2000) desarrolla una síntesis sobre el concepto de evaluación y manifiesta cómo la investigación educativa ha venido posicionando modelos innovadores de enseñanza que dejan ver cuatro rasgos característicos: a) la idea de mejoramiento asociada a la evaluación, donde se evalúa para progresar y no para comprobar, b) la evaluación continua como parte del proceso de enseñanza, de manera que se pueda dar cuenta del aprendizaje en todo momento, c) la evaluación en un sentido global, donde se evalúan actividades, conocimientos y actitudes y d) la evaluación obedece a ritmos personales de aprendizaje y, por tanto, es individual. Esta visión de evaluación tiene un carácter constructivista, en el que se comprende que el estudiante tiene un sistema de ideas que le permite un proceso de explicación y argumentación 
sobre un fenómeno, pero que es susceptible de transformación o deconstrucción.

Sin embargo, esta visión innovadora no siempre se encuentra enla base de las propuestas y desarrollo de evaluaciones en la acción didáctica, así que vale la pena mencionar que, desde una perspectiva en la que se hace énfasis en la transmisión de conocimientos, la evaluación tiene un carácter terminal y de control que se encuentra, a su vez, asociada a los sistemas de prerrequisitos, pues sirve como garante para que se avance de un curso a otro. En este sentido, es una evaluación que lo tiene todo previsto y su incumplimiento revela que los estudiantes no han aprendido. Este concepto de evaluación también ha sido investigado y revisado desde su lógica interna yes coherente con las consideraciones sobre pruebas de entrada que revisan si en verdad los estudiantes tienen los conocimientos para iniciar un curso o un tema. Pero también es coherente con la consideración según la cual estos conocimientos están acumulados y no se relacionan con el tema nuevo en ningún sentido, por tanto, los estudiantes no saben nada del tema que van abordar y sus ideas anteriores sobre otros temas tampoco les servirían de mucho si los siguen aprendiendo en forma desagregada; aun así, se asume aquí la evaluación como un proceso objetivo desde una perspectiva epistemológica positivista.

Para el caso del CDC del profesor de física en formación inicial, se entenderá que este podría estar influenciado por las corrientes de evaluación normativa en el sentido de definir criterios de evaluación, donde los contenidos conceptuales son transversales a las competencias o a los estándares. El criterio fundamental aquí sería la utilización acrítica de los estándares en su organización de valoraciones sobre los aprendizajes de los estudiantes. Una perspectiva usual de la evaluación tradicional (acumulativa, terminal, causal-lineal, trans- misionista) asume a los exámenes, los tests, el quizz, el parcial, la previa, el informe de laboratorio, etc. como actividades de evaluación en sí mismas, aquí no se necesita nada más, ya que el conjunto de estos, o su suma, da cuenta del aprendizaje de los estudiantes. Sin embargo, estas actividades no son más que técnicas de recolección de información sobre las cuales reposan las decisiones terminales de la aprobación o no de una materia. Los criterios asociados a este tipo de visiones sobre la evaluación están condicionados por la idea de transmisión de conocimientos y, por tanto, se basan en el grado de fidelidad que manifiestan los indicios de los datos (la información recolectada) con la verdad de la ciencia. Incluso, hay situaciones de enseñanza en las que se utilizan los mismos instrumentos durante muchos años, pues la supuesta inmutabilidad de los conceptos así lo permite y,en este sentido, las herramientas de evaluación son las mismas actividades, que terminan siendo prueba esencial para certificar los aprendizajes. Así las cosas, algunos criterios asociados con este tipo de evaluación son: a) tareas predefinidas para todos los estudiantes, b) procesos únicos de solución de tareas, ejercicios de lápiz y papel y problemas y c) evaluación sumativa, acumulativa, lineal-causal y terminal.

Ahora bien, desde una perspectiva alternativa, usualmente con referentes constructivistas, la evaluación se entiende como proceso en el que se validan las ideas previas de los estudiantes en un sentido más bien progresivo o de cualificación. La evaluación como proceso involucra tanto la recogida de información como su respectivo análisis, así como la interpretación contextual y la consecuente toma de decisiones que está asociada a la emisión de juicios valorativos sobre la situación o nivel de aprendizaje. Como parte de una propuesta que reconozca la importancia de la evaluación formativa y de la sumativa, Etkina (2002) 
discute algunas actividades de evaluación que pueden ayudar a los estudiantes a centrarse en los aspectos relevantes del trabajo de científicos e ingenieros. La propuesta tiene como fundamento al mensaje que se da a los estudiantes cuando se asumen ciertos tipos de técnicas y actividades de evaluación; por ejemplo, desde un enfoque usual se utilizan exámenes, quizzes e informes de laboratorio, y con esto lo que se le está diciendo al estudiante es que se debe centrar en la comprensión de los conceptos y aprender cómo aplicar ecuaciones en la solución de problemas, así como ser capaz de registrar e interpretar resultados experimentales. Sin embargo, Etkina (2002) advierte que los retos de la vida real son diferentes a los retos organizados y bien definidos de la vida académica. Así, aunque desde esta perspectiva el mensaje sea bueno la relación con el mensaje de la vida real es muy débil. Es decir, un criterio para una evaluación alternativa podría ser el nivel de relación que tienen las actividades de evaluación con los retos de la vida diaria, que se caracterizan principalmente por ser complejos y que para su solución requieren de la construcción misma de problemas, el diseño de investigaciones y productos y el trabajo colaborativo (Etkina, 2002).

Por otro lado, Anderson y Kraftwohl (2001) definen la evaluación como la elaboración de juicios basados en criterios y estándares. A este respecto, Warren (2006) define las estrategias de evaluación como la asociación de criterios, estándares y métodos de aplicación cuando se trata de hacer una valoración sobre procesos o productos en la enseñanza de la física. A su vez, este autor caracteriza las actividades de evaluación formativa desde la perspectiva de Black y William (1998), en la que:

En la evaluación formativa, el profesor tiene que escoger entre dos opciones. La primera es ayudar a desarrollar la capacidad de sus estudiantes para reconocer distinguir todos los vacíos y darles la responsabilidad de planear y llevar a cabo las acciones remediales que puedan necesitar. Esta primera opción implica el desarrollo de la capacidad de autoevaluarse y ayudaren la evaluación colaborativa con otros. La segunda es para que los mismos profesores tomen responsabilidad en la generación de información estimulante y que oriente las actividades subsecuentes (p.23).

Así, uno de los propósitos de la evaluación formativa consiste en generar en los estudiantes y profesores una comprensión sobre lo que se busca, lo que se tiene y las formas de mejorarlo. Para esto, Warren (2006) sugiere el desarrollo de una matriz de valoración en la que se expliciten los diferentes niveles de rendimiento y que puedan ser utilizados por estudiantes y profesores. Esta matriz tiene un carácter organizativo e informativo y puede ser acompañada por comentarios verbales por parte del profesor o del estudiante que conviertan la evaluación en un proceso más personalizado.

La perspectiva de evaluación puede tener también referentes disciplinares muy marcados y asociados con las comprensiones que se acercan mayoritariamente 
al mundo de la física; para este caso, conviene tener elementos que permiten valorar este componente del CDC de los practicantes. En este sentido, es común encontrar la idea de los errores conceptuales de los estudiantes y la literatura investigativa ha venido incursionando de manera que para algunos como Tornkvist, Peterson y Tranströmer (1993) existen errores conceptuales asociados a la comprensión de las trayectorias posibles de una carga en un campo cuando se asocian en su mayoría a las líneas de fuerza. Asimismo, Raduta (2005) señala cómo las falsas pre-concepciones de la mecánica o las analogías que los estudiantes hacen entre campo eléctrico y campo magnético son fuentes de errores conceptuales. La idea de errores conceptuales no discute la posibilidad de que los estudiantes tengan ideas sobre los objetos o fenómenos de la física, es un primer alejamiento de la postura transmisionista, aunque, obviamente, descalifica las concepciones de los estudiantes y generalmente busca reemplazarlas por las verdaderas. Las tareas de evaluación están intrínsecamente asociadas a lo que se pretende lograr en los estudiantes. El grupo PAER (2010) define cinco tipos de tareas de evaluación basadas en un solo principio básico: los estudiantes deben evaluar de alguna manera la solución de un problema propuesto. Los tipos de tareas asociados a la enseñanza de la física son a) evaluación por comparación, b) evaluación por análisis dimensional, c) evaluación por análisis de casos límite-especiales, d) evaluación por análisis de presupuestos y e) evaluación por estimación.

\section{Metodología}

El énfasis de la investigación cualitativa se encuentra en procurar "dar sentido o interpretar los fenómenos en los términos del significado que las personas les otorgan" (Vasilachis, 2006, p. 2), esto quiere decir que en este tipo de investigaciones la interpretación desempeña un rol importante y requiere una fundamentación a partir de categorías o marcos generales. Dado que los trabajos de Strauss y Corbin (2002) destacaron la investigación cualitativa en relación con el estudio no solo del funcionamiento organizacional, sino de "vida de las personas, de historias, de comportamientos" (p. 17), conviene destacar su carácter hermenéutico, propio de los métodos investigativos que buscarían dar cuenta de ello. En particular, en esta tesis se asume la investigación cualitativa basada en la descripción de experiencias y situaciones de enseñanza de la física por parte de profesores practicantes, de manera que será empleada con el fin de caracterizar el CDC de los profesores practicantes procurando "comprender los procesos por los cuales los sucesos y acciones tienen lugar, y desarrollar explicaciones causales válidas analizando cómo determinados sucesos influyen sobre otros" Maxwell (1996, pp. 17-20).Como lo plantean Arnal y La Torre (1992), la finalidad de la investigación interpretativa en educación consiste en "comprender e interpretar la realidad, los significados de las personas, percepciones, intenciones y acciones"(p. 40). Tanto las visiones sobre la relación sujeto-objeto como los criterios de calidad son muy particulares en este tipo de investigación. En cuanto a las primeras, se puede decir que, al contrario de las investigaciones de corte positivista, se entiende como una interrelación en la que el investigador y el investigado se afectan; en cuanto a lo segundo, la calidad se basa en la credibilidad y el poder de transferencia y confirmación de la investigación. 
Tabla 4. Instrumentos de indagación en la fase de planeación

\begin{tabular}{|c|c|}
\hline Instrumento & Descripción general \\
\hline $\begin{array}{l}\text { Encuesta } \\
\text { [ENC] }\end{array}$ & $\begin{array}{l}\text { Un primer punto donde el encuestado debe asignar un orden de importancia que } \\
\text { tienen algunas ideas de la física en relación con el tema del campo eléctrico. } \\
\text { Una pregunta sobre la analogía utilizada por los libros de texto entre el campo } \\
\text { eléctrico y el campo gravitacional. } \\
\text { Un tercer punto en el que al encuestado se le presenta una viñeta de un posible caso } \\
\text { de interpretación de las líneas de fuerza por parte de un estudiante. } \\
\text { Un cuarto punto en el que se solicita al encuestado indicar alternativas frente a las } \\
\text { frecuentes confusiones que presentan los estudiantes entre líneas de fuerza y vector } \\
\text { campo eléctrico. } \\
\text { Un quinto punto en el que se presentan dos situaciones posibles de evaluación de } \\
\text { los estudiantes sobre el tema campo eléctrico y se solicita al encuestado: responder } \\
\text { las dos preguntas como si fuera estudiante, explicar cuáles de estas preguntas } \\
\text { evalúan la comprensión de los estudiantes en términos conceptuales, cuantitativos o } \\
\text { de habilidades científicas y, por último, se le solicita al encuestado indicar si (como } \\
\text { profesor) le haría algunas modificaciones a las preguntas para profundizar los aspectos } \\
\text { de la evaluación anteriormente señalados. La primera de las situaciones se recoge de } \\
\text { la investigación de Furió y Guisasola (2001). }\end{array}$ \\
\hline $\begin{array}{l}\text { Entrevista } \\
\text { [ENT] }\end{array}$ & $\begin{array}{l}\text { Una entrevista semi-estructurada sobre los componentes del PCK que retoma los } \\
\text { presupuestos de Etkina (2010) y que contempla preguntas agrupadas en las siguientes } \\
\text { categorías: } \\
\text { Orientaciones hacia la enseñanza de las ciencias(ideas de los estudiantes, actividades } \\
\text { en clase). } \\
\text { Conocimiento del currículo (contenidos para la enseñanza). } \\
\text { Conocimiento de las dificultades de comprensión de los estudiantes y de las } \\
\text { dificultades con conceptos clave y prácticas de las ciencias (ideas de los estudiantes). } \\
\text { Conocimiento de las estrategias de enseñanza adecuadas para estructurar el } \\
\text { aprendizaje de los estudiantes sobre los conceptos clave y las prácticas de las ciencias } \\
\text { (actividades). } \\
\text { Conocimiento sobre cómo evaluar y acerca del desarrollo de estrategias específicas } \\
\text { para evaluar las comprensiones de los estudiantes de los conceptos clave y las } \\
\text { prácticas de las ciencias (evaluación). }\end{array}$ \\
\hline $\begin{array}{l}\text { El documento } \\
\text { de planeación } \\
{[\mathrm{PA}]}\end{array}$ & $\begin{array}{l}\text { Consiste en el documento que el profesor en formación presenta a su director de la } \\
\text { práctica docente de la universidad. Con este documento, se pretende indagar por } \\
\text { los conocimientos didácticos que el profesor en formación considera adecuados en } \\
\text { la planeación que usualmente se les solicita a los estudiantes del curso de práctica } \\
\text { docente, de acuerdo con los componentes del CDC predefinidos. }\end{array}$ \\
\hline
\end{tabular}

Los instrumentos de indagación (tabla 4) se aplicaron en la primera fase de la investigación, la cual se corresponde con la planeación de la enseñanza del campo eléctrico a estudiantes de un colegio de bachillerato. La encuesta y la entrevista se desarrollaron in situ en la institución y la planeación se solicitó verbalmente al practicante, quien la envió vía correo electrónico al investigador. Las respuestas de los dos primeros instrumentos se transcribieron y se procedió al análisis del contenido en relación con la matriz estilo hipótesis de progresión, entendida como un criterio de organización, secuenciación y jerarquización de los contenidos escolares. En este sentido, García (1998) propone a la hipótesis de progresión en relación con "dimensiones y categorías meta-disciplinares relativas a la transición desde un pensamiento simple hacia otro complejo" (p.154), organización que tiene en cuenta criterios psicológicos, sociológicos y epistemológicos, de manera que "el conocimiento escolar se entiende como un conocimiento organizado y jerarquizado, procesual y relativo, como un sistema de ideas que se reorganiza continuamente en la interacción con otros sistemas de ideas" (García, 1998, p. 151). 
Este enfoque de uso de niveles para "una organización dinámica del conocimiento escolar, mediante propuestas de transición de unos niveles $u$ otros" (p. 151) se utiliza en la presente tesis considerando de manera similar una propuesta estructural de CDC del profesor de física en formación inicial que tiene en cuenta"la utilización de los niveles para una organización dinámica del conocimiento esco- lar, mediante propuestas de transición de unos niveles u otros" (p. 151). Los contenidos de la hipótesis de progresión se organizan entorno a la enseñanza del concepto de campo eléctrico, de manera quela didáctica del campo eléctrico es el eje central. Es decir, cada uno de los niveles de referencia se relaciona con las cuatro categorías del CDC, formando así una estructura de matriz (tabla 5).

Tabla 5. Niveles de Referencia de la Hipótesis de Progresión para la enseñanza del campo eléctrico.

Fuente: Reyes y Martínez, 2011

\begin{tabular}{|c|c|c|c|}
\hline \multicolumn{4}{|c|}{ Niveles de formulación del CDC del profesor de física en formación inicial } \\
\hline $\begin{array}{c}1 \\
\text { (Acrítico) }\end{array}$ & (II & III & $\begin{array}{c}\text { IV } \\
\text { (Reflexivo integral-crítico) }\end{array}$ \\
\hline $\begin{array}{l}\text { Centrado únicamente } \\
\text { en la física, se tienen } \\
\text { referentes que dependen } \\
\text { exclusivamente de la } \\
\text { formación académica } \\
\text { y de los libros de texto. } \\
\text { Se asume la perspectiva } \\
\text { transmisionista desde } \\
\text { una visión acumulativa } \\
\text { lineal tanto de la física } \\
\text { como de la enseñanza. }\end{array}$ & $\begin{array}{l}\text { Centrado en una visión } \\
\text { tecnológica de la enseñanza } \\
\text { caracterizada por una } \\
\text { perspectiva objetivista de la } \\
\text { física que considera algunos } \\
\text { elementos de la normatividad } \\
\text { institucional y un apego por lo } \\
\text { académico los textos, donde se } \\
\text { asumen posturas relacionadas } \\
\text { con el cambio conceptual, los } \\
\text { errores conceptuales. Asimismo, } \\
\text { se consideran los conceptos de } \\
\text { la física desde una perspectiva } \\
\text { elaborada. }\end{array}$ & $\begin{array}{l}\text { Centrado en favorecer un } \\
\text { ambiente de felicidad en } \\
\text { la clase desde un enfoque } \\
\text { espontáneo y a-sistemático, } \\
\text { regulado solamente por la } \\
\text { insatisfacción del profesor } \\
\text { sin manifestar propuestas } \\
\text { fundamentadas en } \\
\text { desarrollos investigativos de } \\
\text { la enseñanza de la física. } \\
\text { Se cuestiona el orden de } \\
\text { los planes de enseñanza, } \\
\text { los libros de texto y se } \\
\text { generan innovaciones desde } \\
\text { la fenomenología para el } \\
\text { estudio de la física. }\end{array}$ & $\begin{array}{l}\text { Se trasciende la reflexión } \\
\text { especulativa dejando a } \\
\text { un lado la perspectiva } \\
\text { transmisionista, se construyen } \\
\text { propuestas fundamentadas } \\
\text { desde perspectivas de } \\
\text { corte investigativo (Porlan, } \\
1998 \text { y Loughran, 2006). } \\
\text { Tanto la física como su } \\
\text { enseñanza se asumen como } \\
\text { constructo desde un enfoque } \\
\text { construccionista. }\end{array}$ \\
\hline
\end{tabular}

\section{Resultados y análisis}

En atención a las limitaciones de espacio, en este artículo se presentan los resultados de la triangulación de los resultados de aplicación de los instrumentos: entrevista [ENT], encuesta [ENC] y planeación [PA], asociados a la matriz de progresión, y se adicionan dos ejemplos metafóricos como mecanismo interpretativo de la información con el propósito de construir la caracterización del CDC. En este proceso, se utilizarán las referencias internas a manera de corchetes cuadrados [n-ENT], [m-ENC], [p-PA], donde $n, m$ y $p$ son las unidades de información correspondientes a la transcripción del material.
En primer lugar, conviene mencionar algunos aspectos del contexto y la historia de vida del profesor practicante. Daniel es un estudiante de 23 años que por primera vez asume el espacio académico de práctica integral en su proceso de formación de la licenciatura en física. Los recuerdos de sus profesores del bachillerato lo llevan a valorar la imagen de su profesora de matemáticas, considerando que su espontaneidad le impactó y le generó un gusto por la profesión, siendo el caso contrario el de su profesora de física, quien, a su juicio, era "...un poquito grosera, como típico profesor" [6-ENT]. Así, ya teniendo que tomar un camino en la universidad, decide estudiar licenciatura en física como una forma de no 
renunciar a sus aspiraciones por la licenciatura en matemáticas y su ideal de "ser un buen profesor". Ahora bien, como él mismo declara, "con el transcurso del tiempo, la física me acogió [...] porque lo pone a uno a pensar más de lo que uno tenía previsto" [4-ENT]. Se sintió entonces con el reto de demostrarse a sí mismo que podía sacar adelante sus estudios en esta carrera. Al comienzo, sintió intereses compartidos con los demás compañeros, pero estos fueron transformándose en maneras de subsistir que obedecían a lógicas menos comunitarias, y ya a la altura de su noveno semestre, todos son de corte individualista. A lo largo de este proceso, pudo recoger una imagen de buen profesor asociada a la distinción de características profesionales, pues ser profesor "no es solo soplar y hacer botellas, un profesor es tener dedicación y tiene que tener estudio" [10-ENT], reconociendo así la importancia de lo que llama "el transcurso de su carrera y de profesión".

En cuanto a su formación en pedagogía y didáctica, Daniel asocia a la primera con mera teoría y lecturas que, en últimas, no le permitían concretar las cosas y en este sentido veía cómo la "pedagogía, pues [...] no servía" [12ENT]. En cuanto a la didáctica, asume que, dado su carácter práctico, asociado a cosas como el uso de libros, los laboratorios y algunos tips, le ha permitido encaminarse mucho más al trabajo de profesor. En este sentido, Daniel considera que los cursos previos a la práctica integral son de teoría de la pedagogía, y asume que en el colegio va a aplicar lo que aprendió en aquellos; sin embargo, la gran diferencia radica en que en los cursos previos su papel era leer y ahora es la "práctica como tal" [2-ENT] asumida como aplicación.

\section{Caracterización del CDC}

Dado que el interés central en esta comunicación se fundamenta en dar cuenta de la caracterización del CDC del profesor practicante (quien en adelante se denominará como Daniel), con respecto a la hipótesis de progresión, se presentan los resultados construidos a partir de la triangulación de la ubicación del CDC en cada categoría en relación con cada uno de los instrumentos. Este resultado global se presenta en la tabla 6.

Tabla 6. Síntesis general de resultados fase de planeaciónen relación con la hipótesis de progresión

\begin{tabular}{|c|c|c|c|c|c|c|c|c|c|c|c|c|c|c|c|c|}
\hline \multirow{2}{*}{$\begin{array}{l}\qquad \text { Nivel } \rightarrow \\
\text { Componentes CDC } \rightarrow \\
\text { Fuentes }\end{array}$} & \multicolumn{4}{|c|}{ I } & \multicolumn{4}{|c|}{ II } & \multicolumn{4}{|c|}{ III } & \multicolumn{4}{|c|}{ IV } \\
\hline & C & I & A & $E$ & C & I & A & $E$ & C & I & A & $E$ & C & I & A & $E$ \\
\hline [ENC] & * & & & $*$ & * & $*$ & $*$ & * & & & & & & & & $*$ \\
\hline [ENT] & * & & & $*$ & * & * & $*$ & & * & & & & & & * & * \\
\hline$[\mathrm{PA}]$ & & * & $*$ & & * & & $*$ & * & & $*$ & $*$ & & $*$ & & & * \\
\hline \multicolumn{17}{|c|}{ *Ubicación del componente en cada nivel de referencia. } \\
\hline \multicolumn{17}{|c|}{ C: Contenidos I: Ideas de los estudiantes A: Actividades E: Evaluación } \\
\hline Encuesta: [ENC] Entrevis & {$[$ EN } & ] 1 & Hed & Sn: & & & & & & & & & & & & \\
\hline
\end{tabular}


El CDC de Daniel manifiesta una mayor frecuencia de referencia en el nivel reflexivo lógico, seguido por el nivel no reflexivo, en contraste con un tercer lugar para el nivel reflexivo integral y por último el nivel innovador. El hecho de que se compartan los dos primeros niveles de referencia indica una tendencia a favorecer una enseñanza de corte más bien tradicional en el sentido del desarrollo de los componentes del CDC. Siendo característico aquí un enfoque de corte transmisionista que le da prioridad a la información y la confunde con conocimiento, y que valida la mirada acumulativa de este, donde se asume también la necesidad de transitar primero por el mundo de la teoría para luego ir a la experimentación y comprobarla.

\section{Confrontar y moldear: el maestro artesano}

Las ideas de los estudiantes son útiles en clase, es por esto que Daniel las reconoce y les imprime una utilidad concreta: confrontarlas con las que él lleva al aula [18-ENT] y poder guiar a sus estudiantes a nuevas ideas, más coherentes, más físicas [7,8-ENC]. Pero el papel de guía involucra tareas específicas en relación con su misión de llevar por buen camino a los estudiantes; en efecto, Daniel se visualiza como un profesor (artesano) que encamina a sus estudiantes moldeando [16ENT] sus modelos mentales, proceso en el que pueden surgir nuevas ideas en los estudiantes, especialmente a la hora de realizar experimentos en clase, frente a lo cual estará atento para desarrollar las preguntas pertinentes.

Una aproximación a este proceso de moldear tiene que ver justamente con el modelo de campo eléctrico que Daniel supone tienen los estudiantes. Aquí, según Daniel, los estudiantes no necesariamente identifican el campo eléctrico con cargas o descargas [18ENT], más bien tendrían una imagen de que el campo es algo que existe en la naturaleza, es decir, asume que sus estudiantes le asignan una ontología al campo. El paso que sigue es fundamental, como se ha mencionado en la sección anterior, pues es con las preguntas e informaciones que Daniel llevará a la clase con lo que se puede comenzar a moldear las ideas de los estudiantes, por eso "tiene uno que empezar a dar los conceptos para que los estudiantes comiencen a preguntar desde ahí"

Figura 4. Representación de la metáfora del maestro artesano

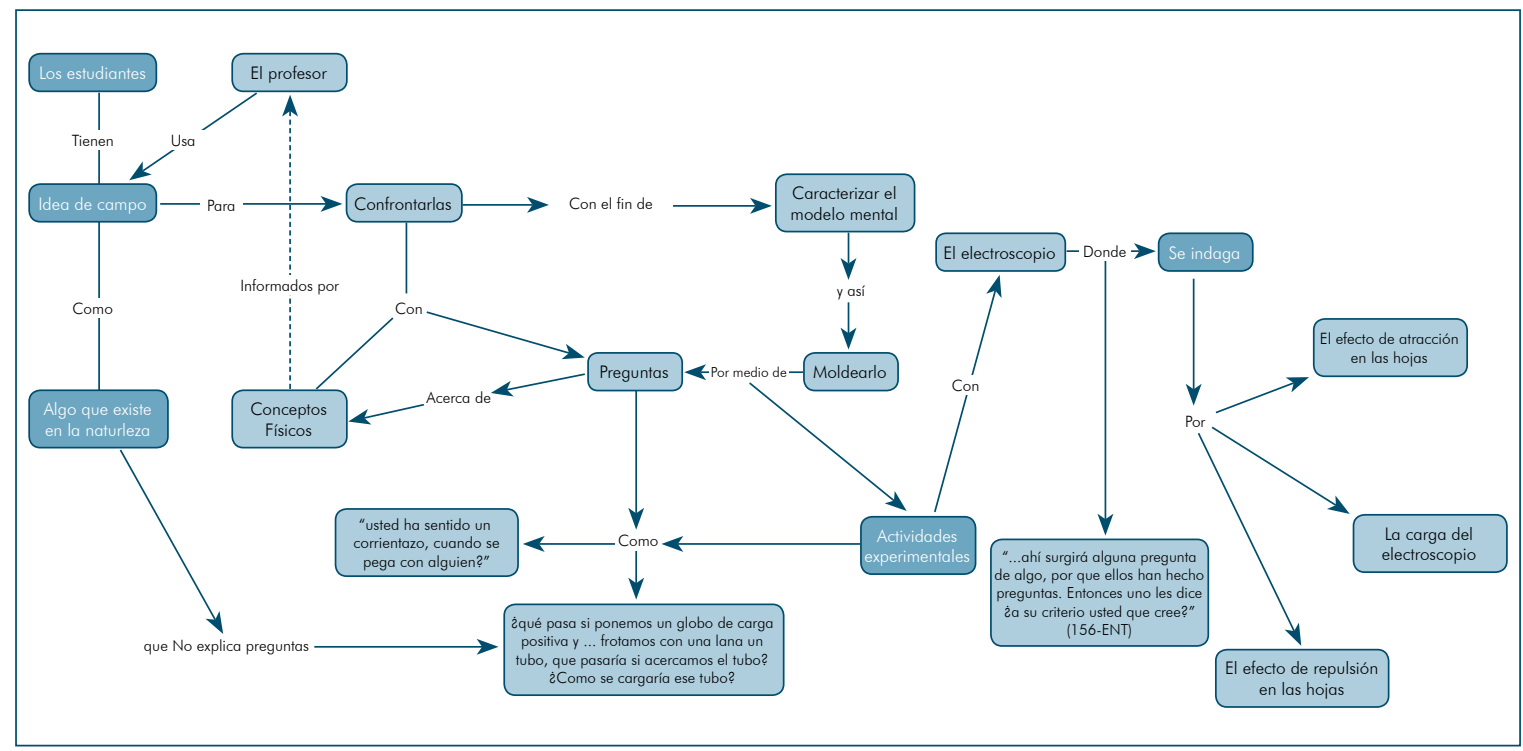


[18-ENT], es decir: la confrontación. Este paso viene acompañado de preguntas y recurrencia a experiencias cotidianas, como el frotamiento de globos o los efectos en el cuerpo cuando se saluda o se rosa con otra persona, es decir, situaciones asociadas a la carga eléctrica. Sin embargo, aquí Daniel asume algunas características del modelo mental de sus estudiantes: el campo eléctrico tiene que ver con la idea de carga eléctrica, los estudiantes no necesariamente distinguen los tipos de carga que producen un campo eléctrico, la frotación produce la carga, no tienen la noción de distribución superficial de la carga eléctrica [26-ENT] y tampoco la idea de campo eléctrico se utiliza para explicar situaciones de inducción eléctrica, como en el caso del globo.

\section{El valor de la contienda}

Daniel también considera que los ejercicios de lápiz y papel-o problemas- son importantes como actividades de clase, pero estos no siempre deben ser problemas cerrados, pues hay que contemplar "unos problemas que sean abiertos y que ellos (los estudiantes) respondan y ataquen desde varios puntos de vista"[30-ENT]. La propuesta de Daniel le implica reflexionar sobre el rol tradicional que han tenido los ejercicios de física en su formación como profesor, ya que asume una crítica a este tipo de actividad cuando se concentra en satisfacer las demandas de Daniel para que quede contento, sobrepasando así las demandas de comprensión de sus estudiantes. En esta dirección, Daniel considera que los ejercicios o problemas deben poner al estudiante a demostrar sus conocimientos y no ser "solo un manejo de números" [30-ENT]. Al parecer, el carácter principal que tendrían este tipo de problemas es permitir un manejo conceptual de la física, en lugar de una manejo netamente matemático.

En este proceso, Daniel se ha venido involucrando en las sesiones iniciales de su práctica docente, especialmente cuando tiene que darle un manejo alternativo a los problemas de lápiz y papel asignados por el profesor tutor, aquí su tarea como profesor practicante es ayudar a los estudiantes a considerar el papel de la fórmula y de los algoritmos como si fuera una lucha o metáfora de contienda entre el estudiante y los ejercicios de lápiz y papel, donde los números parecen ser los representantes de los ejercicios y la autoestima, la autoconfianza y auto-credibilidad representan a los estudiantes. Esta mirada algo animista de la interacción con el conocimiento físico asociado a los procesos de solución de ejercicios se puede relacionar con el enfoque realista comprobatorio asignado a las prácticas experimentales. Así, "atacar la pregunta" [49-ENT] resulta ser una estrategia de la contienda, en la que se debe involucrar una o un grupo de "fórmulas matemáticas" [49-ENT] como insumos o armas que tiene el estudiante, aunado con procesos de motivación que Daniel propone diciendo que invitaría a sus estudiantes a no dejarse amilanar por los comentarios de sus profesores cuando expresan sus ideas [49-ENT]. Esta motivación se encuentra asociada también al desarrollo empático de las relaciones con los estudiantes y como Daniel se concibe como un guía de los estudiantes, podría, seguramente 
ser como el "confidente" [45-ENT], en lugar de asumir el rol de profesor que siempre "da la mala nota" o manipula a sus estudiantes.

\section{Conclusiones}

Dado que no hay una ubicación única en la hipótesis de progresión, el carácter del CDC es más bien polifónico y pone de manifiesto una condición del conocimiento profesional del profesor de física en formación inicial, que consiste en un desarrollo del proceso de transformación didáctica en gestación que se genera a partir de sus primeras experiencias como profesor en una institución educativa.

Para Daniel, los contenidos de enseñanza no difieren mucho de los contenidos de la física per se. Esto permite asegurar que su preocupación se centra en dar fe de su continuidad y la necesidad de asumirlos en forma lineal y acumulativa.

Las actividades aquí reportadas procuran atender el rol pasivo de los estudiantes frente a la solución de ejercicios de lápiz y papel, y el rol de líder de Daniel al plantear estrategias y ayudas en la contienda con los números, las ecuaciones sobre campos eléctricos y las aplicaciones. Este proceso se fundamenta en una visión acumulativa y lineal de las acciones en la clase, de manera que, para llegar a la experimentación, se debe primero pasar por la teoría, proceso que eventualmente deberá culminar con la consecuente evaluación terminal.

\section{Referencias Bibliográficas}

Abell, S. (2008). Twenty years later: does pedagogical content knowledge remain a useful idea? International Journal of Science Education, 30(10), 1405-1416.

Anderson y Kraftwohl, D. (2001). A Taxonomy for learning, teaching, and assessing: a revision of Bloom's taxonomy of educational objectives. Nueva York: Longman.

Arnal, J. del Rincón y La Torre, A. (1992). Investigación educativa: fundamentos y metodología. Barcelona: Lapor.

Arons, A. (1990). A Guide to introductory physics teaching. Nueva York: John Wiley \& Sons Inc.

Bagno, E. (1986). Organization and understanding of concepts in electromagnetism-design implementation and evaluation of an instructional unit for high school. reovot. Israel: Inédito.

Bar, V., Zinn, B. y Rubin, E. (1997). Children's ideas about action at a distance. Doi: 10.1080/0950069970191003.

Bardin, L. (1986). Análisis de contenido. Madrid: Akal.

Barnett, J. y Hodson, D. (2001). Pedagogical context knowledge: Toward a fuller understanding of what good science teachers know. Science Teacher Education, 85, 426-453.

Benejam, P. (1993). Los contenidos de la didáctica de las ciencias sociales en la formación del profesorado. En Montero y Vez (Eds.). Las didácticas específicas en la formación del profesorado (pp. 341 -347). Santiago de Compostela: Tórculo.

Black, P. y William, D. (1998). Inside the black box: raising standards through classroom assessment. Phi Delta Kappan, 80, 139-148. 
Bolívar, A. (1993). Conocimiento didáctico del contenido y formación del profesorado: el programa de L. Shulman. Revista Interuniversitaria de Formación del Profesorado, 16, 113-124.

Carlsen, W. (1999). Domains of teacher knowledge. En J. Gess-Newsome y N. Lederman, Examining pedagogical content knowledge (pp.133-144) Londres: Board.

Erickson, F. (1997). Métodos cualitativos de investigación sobre enseñanza. En M. Wittrock (Comp.), La investigación de la enseñanza, II: Métodos cualitativos y de observación. Madrid: Paidós.

Etkina, E. (2002). Formative and summative assessment in a physics class: Time to change. Recuperado de: http://www.docstoc.com/docs/24441124/Formative-and-summative-assessment-in-a-physics-class-Time

Etkina, E. (2010). Physics pedagogical content knowledge and preparation of high school physics teachers. Physics Education Research, 6, 0201 10-1-0201 10-26.

Furió, C. y Guisasola, J. (1997). Deficiencias epistemológicas de la enseñanza habitual de los conceptos de campo y potencial eléctrico. Enseñanza de las Ciencias, 15 (2), 259-271.

Furió, C. y Guisasola, J. (1998a). Difficulties in learning the concept of electric field. Science Education, 82 (4), 511-526.

Furió, C. y Guisasola, J. (1998b). Dificultades de aprendizaje de los conceptos de carga y campo eléctrico en estudiantes de bachillerato y de universidad. Enseñanza de las Ciencias, 16 (1), 131-146.

Furió C. y Guisasola J. (2001). La enseñanza del concepto de campo eléctrico basada en un modelo de aprendizaje como investigación orientada. Enseñanza De Las Ciencias, 19 (2), 319-334.

García J. (1998). Hacia una teoría alternativa sobre los contenidos escolares. Sevilla: Díada Editora S. L.

Garritz, A., Trinidad R. y Velasco, R. (2004). El conocimiento pedagógico del contenido. Revista Educación Química, 2 (15), 5.

Geli, A. (2000). La evaluación de los procesos y de los resultados en la enseñanza de las ciencias. En Perales y Cañal (Comp.). Didáctica de las ciencias experimentales (pp. 187-206). Alcoy: Marfil.

Grossman, P. (1989) A Study in Contrast: sources in Pedagogical Content Knowledge for secondary English. Journal of Teacher Education, 40, 24-31

Grossman, P. (1990). The making of a teacher. Theacher knowledge and teacher education. Nueva York: Teachers College

Grossman, P., Wilson, S. y Shulman, L. (1989). Teachers of substance:Subject maHer knowledge for teaching. Knowledge base for the beginning teacher. Nueva York: Pergamon Press. 
Gudmundsdottir S. y Shulman, L. (1990). Pedagogical content knowledge in social studies. En Lowyck y Clark (eds.). Teacher thinking and professional action (pp. 23-34). Lewven University Press.

Hewitt, P. (1999). Física conceptual. Serie AWLI. México: Addison Wesley Longman. Pearson.

Loughran, J., Berry, A. y Mulhall, P. (2006). Understanding and developing science teachers' pedagogical content knowledge. Róterdam: Sense Publishers.

Magnusson, S., Krajcik, J. y Borko, H. (1999). Nature, sources, and development of pedagogical content knowledge for science teaching. En J. Gess-Newsome y N. Lederman (Eds.). Examining pedagogical content knowledge. The construct and its implications for science education (pp. 95-132). Londres: Kluwer Academic Publishers.

Marcelo, C. (1992). Como conocen los profesores la materia que enseñan. Algunas contribuciones de la investigación sobre Conocimiento Didáctico del Contenido. Las Didácticas específicas en la Formación del profesorado. Recuperado de http://ocw.pucv.cl/cursos-1/epe 1 137/materiales-de-clases-1/unidad-2/construccion-conocimiento-profesional

Marks, R. (1990). Pedagogical content knowledge: from a mathematical case to a modified conception. Journal of Teacher Education, 41 (3), 3-1 1.

Martin del Pozo, R. y Rivero, A. (2001). Construyendo un conocimiento profesionalizado para enseñar ciencias en la educación secundaria: los ámbitos de investigación profesional en la formación inicial del profesorado. Revista Interuniversitaria de Formación del Profesorado, 40, 63-79.

Martín, J. y Solbes, J. (2001). Diseño y evaluación de una propuesta para la enseñanza del concepto de campo. Revista Enseñanza de las Ciencias, 19 (3), 393-40.

Maxwell, J. (1996). Qualitative research desings. An interactive approach. Sage Publications, $1-13$.

Morales I. e Infante, E. (2005). Física 2. Bogotá: Editorial Norma.

Morine-Dershimer, G. y Kent, T. (1999). The complex nature and sources of teachers' pedagogical content knowledge. En Gess-Newsome y Lederman (Eds.). Examining pedagogical content knowledge. The construct and its implications for science education (pp. 21 -50). Londres: Dordrecht.

Perafán, G. (2004). La epistemología del profesor sobre su propio conocimiento profesional. Tesis Doctoral. Universidad Pedagógica Nacional, Bogotá, Colombia.

Pomer, F. (1994). Electromagnetisme basic. Valencia: Universidad de Valencia.

Poon, C. (1986). Teaching field concept and potential energy at A-level. Physics Education, 21, 307-316.

Raduta, C. (2005). General students misconceptions related to electricity and magnetism. Ohio: Physics Department of the Ohio State University. 
Reyes, J. (2010). Tendencias en investigación en el conocimiento pedagógico de contenido de profesores de física en formación inicial. Revista de Enseñanza de la Física, 23 (1-2), 7-19.

Reyes, J. y Martínez, C. (2011). Conocimiento didáctico del contenido en profesores de física en formación inicial. Revista Tecné, Episteme y Didaxis: TED, número Extraordinario.

Rutgers University Physics and Astronomy Education Research (2010). Assesment tasks rutgers university. Recuperado de: http://paer.rutgers.edu/ScientificAbilities/ Formative+Assessment+Tasks/default.aspx

Shulman, L. (1986). Those who understand: knowledge growth in teaching. Educational Researcher, 15 (2), 4-14.

Segall, A. (2004). Revisiting pedagogical content knowledge: the pedagogy of content/the content of pedagogy. Teaching and Teacher Education, 20 (5), 489-504.

Strauss, A. y Corbin, J. (2002). Bases de la investigación cualitativa. Técnicas y procedimientos para desarrollar la teoría fundamentada. (2da. Ed.). Bogotá, Colombia: CONTUS-Editorial Universidad de Antioquia.

Törnkvist, K., Peterson A, y Tranströmer, G. (1993). Confusion by representation: On student's comprehension of the electric field concept. American Journal of Physics, 61 (4), 335-338.

Valbuena, E. (2007). El conocimiento didáctico de contenido biológico. Estudio de las concepciones disciplinares y didácticas de futuros docentes de la Universidad Pedagógica Nacional (UPN). Tesis Doctoral. Universidad Complutense de Madrid. Madrid, España.

Van Driel, J. y Verloop, N. (1999). Teachers' knowledge of models and modeling in science. International Journal of Science Education, 21 (11), $1141-1153$.

Vasilachis, I. (2006). Estrategias de investigación cualitativa. Barcelona: Gedisa.

Viennot, L. (2002). Raisonnement à plusieurs variables: tendances de la pensée commune, Aster, 14, 127-141.

Viennot, L. y Rainson, S. (1992). Students' reasoning about the superposition of electric field. International Journal of Science Education, 14 (4), 475-487.

Villegas, R.y Ramírez, R. (1998). Galaxia Física 11. Bogotá: Voluntad.

Warren, A. (2006). Evaluation strategies as a means for learning physics. Recuperado de: http://paer.rutgers.edu/ScientificAbilities/Papers + Talks + and + Presentations/ default.aspx

Wilson, D. y Buffa, J. (2003). Física. México: Pearson.

Zalamea, E., Rodríguez, J. y Paris, R. (2001). Guía Física 11 . Bogotá: Educar Editores.

Zitzewitz, P., Neff, R. y Davids, M. (1995). Física Principios y Problemas 2. Bogotá: Mc Graw Hill. 